EESTI NSV TEADUSTE AKADEEMIA TOIMETISED. XII KÖIDE FOOSIKA-MATEMAATIKA- JA TEHNIKATEADUSTE SEERIA. 1963, NR. 3

ИЗВЕСТИЯ АКАДЕМИИ НАУК ЭСТОНСКОИ ССР. ТОМ ХІІ СЕРИЯ ФИЗИКО-МАТЕМАТИЧЕСКИХ И ТЕХНИЧЕСКИХ НАУК. 1963, № 3

\title{
ОБ ИНТЕРПОЛЯЦИОННОМ АНАЛОГЕ МЕТОДА ГРАДИЕНТОВ
}

\author{
С. Ульм, \\ кандидат физико-математнческих наук
}

Для приближенного решения нелинейного операторного уравнения

$$
P(x)=0
$$

в вещественном гильбертовом пространстве $H$ метод градиентов может быть получен следующим образом (ср. [5,6]):

заменим уравнение (1) эквивалентным ему функциональным уравнением

Так как

$$
\Phi(x) \equiv\|P(x)\|^{2}=0 .
$$

$$
\operatorname{grad} \Phi(x)=2\left[P^{\prime}(x)\right]^{*} P(x),
$$

то, исходя из приближения $x_{n}$ к решению $x^{*}$ уравнения (1), следующее приближенне $x_{n+1}$ выбирается в направлении антиградиента

$$
\begin{gathered}
x_{n+1}=x_{n}-\varepsilon\left[P^{\prime}\left(x_{n}\right)\right]^{*} P\left(x_{n}\right), \\
(n=0,1, \ldots)
\end{gathered}
$$

причем $\varepsilon-$ некоторое положительное число. ${ }^{*}$

В данной заметке мы используем понятие аналога разделенных разностей $F\left(x^{\prime}, x^{\prime \prime}\right)$ для функционала $F(x)$ (см. $\left.\left[{ }^{3}\right]\right)$. Так как в гильбертовом пространстве по определению [']

$$
F^{\prime}(x) h=(h, \operatorname{grad} F(x)),
$$

то аналогично (5) определим и понятие интерполяционного аналога градиента для функционала $F(x)$, который обозначим через $\tilde{F}\left(x^{\prime}, x^{\prime \prime}\right)$ :

$$
F\left(x^{\prime}, x^{\prime \prime}\right) h=\left(h, \tilde{F}\left(x^{\prime}, x^{\prime \prime}\right)\right) .
$$

Для функционала $\Phi(x)=\|P(x)\|^{2}$ легко получается

откуда

$$
\Phi\left(x^{\prime}, x^{\prime \prime}\right) h=\left(P\left(x^{\prime}, x^{\prime \prime}\right) h, P\left(x^{\prime}\right)+P\left(x^{\prime \prime}\right)\right),
$$

$$
\tilde{\Phi}\left(x^{\prime}, x^{\prime \prime}\right)=P^{*}\left(x^{\prime}, x^{\prime \prime}\right)\left[P\left(x^{\prime}\right)+P\left(x^{\prime \prime}\right)\right],
$$

где $P^{*}\left(x^{\prime}, x^{\prime \prime}\right)$ - сопряженный к $P\left(x^{\prime}, x^{\prime \prime}\right)$ оператор.

- Вместо фиксированного $\varepsilon$ в (4) рассматривается и последовательность положи тельных чисел $\left\{\varepsilon_{n}\right\}$ (см. $\left.[2,5,6]\right)$. 
Исходя из приближений $x_{n}, x_{n-1}$ и выбирая следующее приближение в направлении интерполяционного аналога антиградиента, получим итерационный метод

$$
\begin{gathered}
x_{n+1}=x_{n}-\varepsilon P^{*}\left(x_{n}, x_{n-1}\right)\left[P\left(x_{n}\right)+P\left(x_{n-1}\right)\right], \\
\varepsilon>0 ; n=0,1, \ldots
\end{gathered}
$$

Ниже доказываются две теоремы о сходимости метода (9). Прежде всего покажем, что справедлива следующая

Л емм а. Пусть

$$
\begin{aligned}
\eta_{n+1} & \leqslant \sqrt{A_{1} \eta_{n}^{2}+A_{2} \eta_{n-1}^{2}+A_{3} \eta_{n-2}^{2}+A_{4} \eta_{n} \eta_{n-1}+A_{5} \eta_{n-1} \eta_{n-2}}+ \\
& +B_{1} \eta_{n}^{2}+B_{2} \eta_{n-1}^{2}+B_{3} \eta_{n} \eta_{n-1}+B_{4} \eta_{n} \eta_{n-2}+B_{5} \eta_{n-1} \eta_{n-2},
\end{aligned}
$$

причем $A_{i} \geqslant 0, B_{i} \geqslant 0(i=1, \ldots, 5)$ и $. \eta_{t} \geqslant 0(i=-1,0,1, \ldots)$. Если

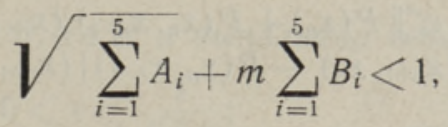

где $m=\max \left(\eta_{-1}, \eta_{0}, \eta_{1}\right)$, то существует

$$
\lim _{n \rightarrow \infty} \eta_{n}=0 \text {. }
$$

Доказательство. По индукции легко установить, что $\eta_{n}<m$ $(n=2,3, \ldots)$. Отсюда вытекает существование

$$
\varlimsup_{n \rightarrow \infty} \eta_{n}=c, \text { причем } 0 \leqslant c \leqslant m .
$$

Допустим, что $c \neq 0$. Переходя к верхнему пределу в неравенствах (10), получаем противоречие:

$$
c \leqslant c \sqrt{\sum_{i=1}^{5} A_{i}+c^{2} \sum_{i=1}^{5} B_{i}<c .}
$$

Итак, $\overline{\lim } \eta_{n}=0$. Так как $\eta_{n} \geqslant 0$, то существует $\lim \eta_{n}=0$. Лемма до$n \rightarrow \infty$ казана.

Те орем а 1. Пусть в замкнутой области $Q$, содержащей последовательность $\left\{x_{n}\right\}(n=-1,0, \ldots)$ справедливы оценки
a) $\left\|P^{*}\left(x^{\prime}, x^{\prime \prime}\right) h\right\| \geqslant \frac{1}{\sqrt{M}}\|h\|, \quad 0<M<+\infty$,
б) $\left\|P\left(x^{\prime}, x^{\prime \prime}\right)\right\| \leqslant \sqrt{K}$
в) $\left\|P\left(x^{\prime}, x^{\prime \prime}\right)-P\left(x^{\prime \prime}, x^{\prime \prime \prime}\right)\right\| \leqslant L\left\|x^{\prime}-x^{\prime \prime \prime}\right\|$.

длякаждого $x^{\prime}, x^{\prime \prime}, x^{\prime \prime} \in Q, h \in H$ и выполнено одно из условий $1^{\circ}, 2^{\circ}, 3^{\circ}$ :

$1^{\circ} \quad M K \leqslant a ; 0<\varepsilon \leqslant \frac{3-\sqrt{9-4 M^{2} K^{2}}}{2 M K^{2}} ; \quad \sqrt{3 K^{3} \varepsilon^{3}+3 K^{2} \varepsilon^{2}-\frac{3}{M} \varepsilon+1}+$

$$
+8 K L m \varepsilon^{2}<1
$$


$2^{\circ} \quad M K \leqslant a ; \varepsilon>\frac{3-\sqrt{9-4 M^{2} K^{2}}}{2 M K^{2}} ; \quad \sqrt{3 K^{3} \varepsilon^{3}+2 K^{2} \varepsilon^{2}}+8 K L m \varepsilon^{2}<1 ;$

$3^{\circ} \quad M K \geqslant a ; \sqrt{3 K^{3} \varepsilon^{3}+3 K^{2} \varepsilon^{2}-\frac{3}{M} \varepsilon+1}+8 K L m \varepsilon^{2}<1$;

где $а$ - корень уравнения $2 x^{3}+5 x^{2}-2 x-9=0 \quad(a \approx 1,239) \quad u$ $m=\max \left\{\left\|P\left(x_{-1}\right)\right\| ;\left\|P\left(x_{0}\right)\right\| ;\left\|P\left(x_{1}\right)\right\|\right\}$.

Тогда уравнение (1) имеет в области $Q$ единственное решение $x^{*}$, $\kappa$ которому последовательность (9) сходится.

Д ок а а тельство. Введем обозначения:

$$
\left\|P\left(x_{i}\right)\right\|=\eta_{i} \quad(i=-1,0, \ldots) .
$$

На основании интерполяционной формулы Ньютона [3]

$$
\begin{aligned}
& \left\|P\left(x_{n+1}\right)\right\| \leqslant\left\|P\left(x_{n}\right)+P\left(x_{n}, x_{n-1}\right)\left(x_{n+1}-x_{n}\right)\right\|+ \\
& \quad+\left\|\left[P\left(x_{n+1}, x_{n}\right)-P\left(x_{n}, x_{n-1}\right)\right]\left(x_{n+1}-x_{n}\right)\right\| .
\end{aligned}
$$

Для $n=1,2, \ldots$ получим

$$
\begin{gathered}
\left\|P\left(x_{n}\right)+P\left(x_{n}, x_{n-1}\right) \quad\left(x_{n+1}-x_{n}\right)\right\|^{2}= \\
=\left\|P\left(x_{n}\right)-\varepsilon P\left(x_{n}, x_{n-1}\right) P^{*}\left(x_{n}, x_{n-1}\right)\left[P\left(x_{n}\right)+P\left(x_{n-1}\right)\right]\right\|^{2}= \\
=\left\|P\left(x_{n}\right)\right\|^{2}-2 \varepsilon\left\|P^{*}\left(x_{n}, x_{n-1}\right) P\left(x_{n}\right)\right\|^{2}- \\
-2 \varepsilon\left(P\left(x_{n}, x_{n-1}\right) P^{*}\left(x_{n}, x_{n-1}\right) P\left(x_{n}\right), P\left(x_{n-1}\right)\right)+ \\
+\varepsilon^{2}\left\|P\left(x_{n}, x_{n-1}\right) P^{*}\left(x_{n}, x_{n-1}\right)\left[P\left(x_{n}\right)+P\left(x_{n-1}\right)\right]\right\|^{2}= \\
=\left\|P\left(x_{n}\right)\right\|^{2}-3 \varepsilon\left\|P^{*}\left(x_{n}, x_{n-1}\right) P\left(x_{n}\right)\right\|^{2}-\varepsilon\left\|P^{*}\left(x_{n}, x_{n-1}\right) P\left(x_{n-1}\right)\right\|^{2}+ \\
+\varepsilon\left\|P^{*}\left(x_{n}, x_{n-1}\right)\left[P\left(x_{n}\right)-P\left(x_{n-1}\right)\right]\right\|^{2}+ \\
+\varepsilon^{2}\left\|P\left(x_{n}, x_{n-1}\right) P^{*}\left(x_{n}, x_{n-1}\right)\left[P\left(x_{n}\right)+P\left(x_{n-1}\right)\right]\right\|^{2} .
\end{gathered}
$$

Поскольку

$\left\|P^{*}\left(x_{n}, x_{n-1}\right)\left[P\left(x_{n}\right)-P\left(x_{n-1}\right)\right]\right\|=\left\|P^{*}\left(x_{n}, x_{n-1}\right) P\left(x_{n}, x_{n-1}\right)\left(x_{n}-x_{n-1}\right)\right\|=$ $=\varepsilon\left\|P^{*}\left(x_{n}, x_{n-1}\right) P\left(x_{n}, x_{n-1}\right) P^{*}\left(x_{n-1}, x_{n-2}\right)\left[P\left(x_{n-1}\right)+P\left(x_{n-2}\right)\right]\right\|$,

10 на основании оценок а и б

$$
\begin{gathered}
\left\|P\left(x_{n}\right)+P\left(x_{n}, x_{n-1}\right)\left(x_{n+1}-x_{n}\right)\right\|^{2} \leqslant \\
\leqslant\left(K^{2} \varepsilon^{2}-\frac{3}{M} \varepsilon+1\right) \eta_{n}^{2}+\left(K^{3} \varepsilon^{3}+K^{2} \varepsilon^{2}-\frac{1}{M} \varepsilon\right) \eta_{n-1}^{2}+ \\
+K^{3} \varepsilon^{3} \eta_{n_{i}-2 j}^{2}+2 K^{2} \varepsilon^{2} \eta_{n} \eta_{n-1}+2 K^{3} \cdot \varepsilon^{3} \eta_{n-1} \eta_{n-2} .
\end{gathered}
$$

Так как

$$
\begin{gathered}
\left\|\left[P\left(x_{n+1}, x_{n}\right)-P\left(x_{n}, x_{n-1}\right)\right]\left(x_{n+1}-x_{n}\right)\right\| \leqslant L\left\|x_{n+1}-x_{n}\right\|\left\|x_{n+1}-x_{n-1}\right\| \leqslant \\
\leqslant L\left\|x_{n+1}-x_{n}\right\|\left(\left\|x_{n+1}-x_{n}\right\|+\left\|x_{n}-x_{n-1}\right\|\right) \leqslant \\
\leqslant K L \varepsilon^{2}\left(\eta_{n}^{2}+2 \eta_{n-1}^{2}+3 \eta_{n} \eta_{n-1}+\eta_{n} \eta_{n-2}+\eta_{n-1} \eta_{n-2}\right),
\end{gathered}
$$

то из $(13),(16)$ и (17) вытекает

$$
\begin{aligned}
& \eta_{n+1} \leqslant\left[\left(K^{2} \varepsilon^{2}-\frac{3 \varepsilon}{M}+1\right) \eta_{n}^{2}+\left(K^{3} \varepsilon^{3}+K^{2} \varepsilon^{2}-\frac{1}{M} \varepsilon\right) \eta_{n-1}^{2}+\right. \\
& \left.\quad+K^{3} \varepsilon^{3} \eta_{n-2}^{2}+2 K^{2} \varepsilon^{2} \eta_{n} \eta_{n-1}+2 K^{3} \varepsilon^{3} \eta_{n-1} \eta_{n-2}\right]^{1 / 2}+ \\
& +K L \varepsilon^{2}\left(\eta_{n}^{2}+2 \eta_{n-1}^{2}+3 \eta_{n} \eta_{n-1}+\eta_{n} \eta_{n-2}+\eta_{n-1} \eta_{n-2}\right) .
\end{aligned}
$$


Используя доказанную лемму, нетрудно установить, что при выполнении одного из условий $1^{\circ}, 2^{\circ}$ и $3^{\circ}$

$$
\lim _{n \rightarrow \infty} \| P\left(x_{n}\right)=\lim _{n \rightarrow \infty} \eta_{n}=0
$$

Единственность решения в замкнутой области $Q$ вытекает из условия а (ср. $\left.\left.{ }^{4}\right]\right)$.

Теорема доказана.

Теорем а 2. Пусть в замкнутой области $Q$, содержащей последовательность $\left\{x_{n}\right\}(n=-1,0, \ldots)$, справедливы оценки

a) $\left(P\left(x^{\prime}, x^{\prime \prime}\right) P^{*}\left(x^{\prime \prime}, x^{\prime \prime \prime}\right) h, h\right) \geqslant \frac{1}{M}\|h\|^{2}, 0<M<+\infty$,

б) $\left\|P\left(x^{\prime}, x^{\prime \prime}\right)\right\| \leqslant \sqrt{K}$

для каждого $x^{\prime}, x^{\prime \prime}, x^{\prime \prime \prime} \in Q, h \in H$ и выполнено одно из условий $1^{\circ}, 2^{\circ}$ :

$$
\begin{array}{ll}
1^{\circ} M K<\frac{\sqrt{7}}{2} ; & 0<\varepsilon<\frac{1}{\sqrt{7} K} ; \\
2^{\circ} M K \geqslant \frac{\sqrt{7}}{2} ; & 0<\varepsilon<\frac{1}{2 M K^{2}} .
\end{array}
$$

Тогда уравнение (1) имеет в области $Q$ единственное решение $x^{*}$. * которому последовательность (9) сходится.

Д оказательство. Исходя из равенства

$$
P\left(x_{n+1}, x_{n}\right)\left(x_{n+1}-x_{n}\right)=P\left(x_{n+1}\right)-P\left(x_{n}\right),
$$

получим для $n=1,2, \ldots$

$$
\begin{gathered}
\left\|P\left(x_{n+1}\right)\right\|^{2}=\left\|P\left(x_{n}\right)+P\left(x_{n+1}, x_{n}\right)\left(x_{n+1}-x_{n}\right)\right\|^{2}= \\
=\left\|P\left(x_{n}\right)\right\|^{2}-2 \varepsilon\left(P\left(x_{n+1}, x_{n}\right) P^{*}\left(x_{n}, x_{n-1}\right)\left[P\left(x_{n}\right)+P\left(x_{n-1}\right)\right], P\left(x_{n}\right)\right)-1 \\
+\varepsilon^{2}\left\|P\left(x_{n+1}, x_{n}\right) P^{*}\left(x_{n}, x_{n-1}\right)\left[P\left(x_{n}\right)+P\left(x_{n-1}\right)\right]\right\|^{2}= \\
=\left\|P\left(x_{n}\right)\right\|^{2}-4 \varepsilon\left(P\left(x_{n+1}, x_{n}\right) P^{*}\left(x_{n}, x_{n-1}\right) P\left(x_{n}\right), P\left(x_{n}\right)\right)+ \\
+2 \varepsilon\left(P\left(x_{n+1}, x_{n}\right) P^{*}\left(x_{n}, x_{n-1}\right)\left[P\left(x_{n}\right)-P\left(x_{n-1}\right)\right], P\left(x_{n}\right)\right)+ \\
+\varepsilon^{2}\left\|P\left(x_{n+1}, x_{n}\right) P^{*}\left(x_{n}, x_{n-1}\right)\left[P\left(x_{n}\right)+P\left(x_{n-1}\right)\right]\right\|^{2}
\end{gathered}
$$

Так как

$$
\begin{gathered}
P\left(x_{n}\right)-P\left(x_{n-1}\right)=P\left(x_{n}, x_{n-1}\right)\left(x_{n}-x_{n-1}\right)= \\
=-\varepsilon P\left(x_{n}, x_{n-1}\right) P^{*}\left(x_{n-1}, x_{n-2}\right)\left[P\left(x_{n-1}\right)+P\left(x_{n-2}\right)\right],
\end{gathered}
$$

то, обозначив $\left\|P\left(x_{i}\right)\right\|=\eta_{i}$, получим на основании (20) и (21)

$$
\begin{aligned}
\eta_{n+1}^{2} \leqslant & \left(K^{2} \varepsilon^{2}-\frac{4}{M} \varepsilon+1\right) \eta_{n}^{2}+K^{2} \varepsilon^{2} \eta_{n-1}^{2}+ \\
& +4 \varepsilon^{2} K^{2} \eta_{n} \eta_{n-1}+2 \varepsilon^{2} K^{2} \eta_{n} \eta_{n-2}
\end{aligned}
$$

Теперь нетрудно установить (ср. с доказательством теоремы 1), что условие $1^{\circ}$ (или $2^{\circ}$ ) обеспечивает сходимость последовательности (9) $k$ решению $x^{*}$. Единственность решения опять-же вытекает из условия а (ср. $\left.\left[{ }^{4}\right]\right)$.

Теорема доказана.

2 ENSV TA Toimetised T-3 1963 
3 а мечани е. Теоремы 1 и 2 остаются в силе, если в (9) $\varepsilon$ заменить $\varepsilon_{n}$, причем последовательность положительных чисел $\left\{\varepsilon_{n}\right\}$ неубывающая. В формулировках теорем заменим $\varepsilon$ числом sup $\varepsilon_{n}$.

При приближенном решении линейного операторного уравнения

$$
T x \equiv A x+b=0 ; b \in H
$$

метод (9) принимает вид

$$
\begin{gathered}
x_{n+1}=x_{n}-\varepsilon A^{*}\left(T x_{n}+T x_{n-1}\right) . \\
(\varepsilon>0)
\end{gathered}
$$

Так как в данном случае $L=0$, то из теоремы 1 следует

Т е о рем а 3: Пусть справедливы оценки
a) $\left\|A^{*} h\right\| \geqslant \frac{1}{\sqrt{M}}\|h\|$,
$0<M<+\infty, h \in H$,
б) $\|A\| \leqslant \sqrt{K}$

и выполнено одно из условий $1^{\circ}, 2^{\circ}$ :

$$
\begin{array}{ll}
1^{\circ} M K \leqslant a ; & 3 K^{3} \varepsilon^{3}+2 K^{2} \varepsilon^{2}<1 \\
2^{\circ} M K \geqslant a ; & \varepsilon<\frac{\sqrt{M^{2} K^{2}+4 M K}-M K}{2 M K^{2}}
\end{array}
$$

где $а$ - корень уравнения $2 x^{3}+5 x^{2}-2 x-9=0(a \approx 1,239)$.

Тогда последовательность (24) сходится к решению уравнения (23).

\section{ЛІ И Т Е Р А Т У Р А}

1. M. М. В а й н бе р г, Вариационные методы исследования нелинейных операторов, M., 1956.

2. Е. И. Л и н ь ко в, О методе наискорейшего спуска для нелинейных уравнений, Ученые записки Московск. обл. пед. ин-та, т. XCVI, 6, 1960, 221-230.

3. А. С. С е р ге ев, О методе хорд, Сибирский матем. журнал, т. II, 2, 1961, 282-289.

4. С. У льм, Об одном классе итерационных методов в пространстве Гильберта, Изв. АН ЭССР, Сер. физ.-матем. и техн. наук, т. XII, № 2, 1963.

5. В. М. Фр и дм а н, Итеративный процесс с минимальными ошибками для нелинейного операторного уравнения, ДАН, т. 139, № 5, 1961, 1063-1066.

6. M. Altman, Concerning approximate solutions of non-linear functional equations, Bull. Acad. Polon. Sci., Cl. 3, 5, № 5, 1957, 460-465.

Институт кибернетики

Академии наук Эстонской ССР
Поступила в редакцию

9. II 1963 


\section{GRADIENDIMEETODI INTERPOLATSIOONANALOOG}

S. Ulm,

füüsika-matemaatikateaduste kandidaat

\section{Resümee}

Defineeritakse funktsionaali gradiendi interpolatsioonanaloogi mōiste (valem 6), millest lähtudes vaadeldakse Hilberti ruumis iteratsioonimeetodit (9). Teoreemidega 1 ja 2 antakse piisavad tingimused, milledel jada (9) koondub mittelineaarse operaatorvôrrandi (1) lahendiks. Erijuhuna vaadeldakse meetodi rakendamist lineaarsele operaatorvōrrandile (teoreem 3).

Eesti NSV Teaduste Akadeemia Küberneetika Instituut

\section{Saabus toimetusse}

9. II 1963

\section{OBER DAS INTERPOLATIONSANALOGON DER GRADIENTMETHODE}

\section{S. UIm}

\section{Zusammenfassung}

Es wird ein Interpolationsanalogon des Gradienten für ein Funktional definiert (Formel 6) und zur Behandlung der Iterationsmethode (9) im hilbertschen Raum verwendet. Die Theoreme 1 und 2 bestimmen hinreichende Bedingungen für die Konvergenz der Folge (9) zur Lösung einer nichtlinearen Operatorgleichung (1). Als ein Spezialfall wird die Anwendung der Methode für eine lineare Operatorgleichung behandelt (Theorem 3).

Institut für Kybernetik

der Akademie der Wissepschaften der Estnischen SSR
Eingegangen

am 9. Febr. 1963 